\title{
Unexpected discrimination strategy used by pigeons
}

\author{
Lorenzo von Fersen ${ }^{a}$, Jacky Emmerton ${ }^{b}$ and Juan D. Delius ${ }^{a}$ \\ "Allgemeine Psychologie, Universität Konstanz, Konstanz, FRG, and ${ }^{b}$ Department \\ of Psychological Sciences, Purdue University, West Lafayette, Indiana, USA
}

\begin{abstract}
Pigeons were conditioned with a symbolic matching-to-sample paradigm. Six visual patterns in 16 configurations were presented in an operant chamber where reinforcement was delivered next to the correct keys. A test with novel configurations, planned to demonstrate associative transitivity between equivalent stimuli, revealed instead a different but consistent behavior. An explanation for this behavior was sought in further tests and in a detailed post-hoc analysis of the training configuration. The analysis revealed an overall location imbalance of the comparison stimuli in relation to the reinforcement sites. This apparently led to response side biases controlled by these lateral stimuli. The results of additional tests accorded with this hypothesis. The directing effect was unequivocal when both lateral stimuli biased a response in the same direction. When the directing effects by these stimuli were opposite, the animals only solved the task when they could resort to a rote learning of individual configurations. The characteristics of the rules that were identified are contrasted with those that have previously been thought to operate during conditional discrimination learning.
\end{abstract}

Key words: Conditional discrimination; Symbolic matching-to-sample; Stimulus control; Stimulus-response rules; Side bias; Pigeon

\section{Introduction}

In recent years the conditional discrimination procedure known as riatching-to-sample has become a much employed paradigm. Several variants of it lend themselves to the study

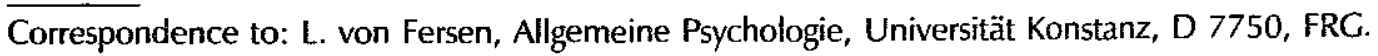


of various aspects of arimal cognition. This paper is concerned with unusual results obtained with one modification, the so-called symbolic matching-to-sample procedure (Carter and Werner, 1978). In the most usual form of this paradigm, subjects are successively presented with a series of three-stimulus configurations, each assembled from the same small set of different stimuli. The middle stimulus of each such configuration serves as a sample, the side stimuli as comparisons. One of the comparison stimuli constitutes a so-called matching pair with the sample stimulus, the other does not. If the subject chooses the former comparison it is rewarded, if it chooses the latter one it is punished. The experimenter determines which particular sample-comparison stimulus pairings serve as matching pairs and which do not. The task is said to be of the symbolic type when the stimuli making up the matching pairs do not resemble each other physically.

The intention of the initial experiment we describe was to establish whether pigeons that had learned to treat certain such stimuli as equivalent (symbolically matching) would transfer that equivalence to a novel pairing of the same stimuli (transitivity; Sidman, 1990 ). Given that pigeons had learned that the stimulus doublets $\mathrm{A}-\mathrm{B}$ and $\mathrm{B}-\mathrm{C}$ were two matching pairs in the above sense, the initial question was whether they would then respond to the novel doublet A-C as if it was a matching pair ton. As will be described, our pigeons did not in fact evince such transitive behavior. Instead, they showed a highly consistent response pattern that defied immediate interpretation. A series of tests was performed to investigate the reasons for this response consistency. These tests ultimately revealed that, although apparently successfully condition $\perp$ to solve symbolic matching-to-sample tasks, our pigeons behaved according to a strategy not previously considered in connection with this paradigm (Carter and Werner, 1978). The results suggest that spatial contingencies may play a more important role in determining choice behavior in complex discriminatory paradigms than is generally acknowledged.

\section{Experiment I}

\section{Method}

\section{Subjects and apparatus}

Six experimentally naive adult homing pigeons (Columba livia) of local origin were used. During the experiment they were maintained at $80 \%$ of their normal body weights. A chambe $(33 \times 34 \times 33 \mathrm{~cm})$ incorporating an alcove $(16 \times 14 \times 10 \mathrm{~cm})$ was employed. The floor of the alcove (Fig. 1) contained three horizontally positioned response keys. The keys, made of a translucent plastic, were $2.2 \mathrm{~cm}$ in diameter and $4.5 \mathrm{~cm}$ apart (center-tocenter). Three separate food cups $(1.5 \mathrm{~cm}$ in diameter, $0.5 \mathrm{~cm}$ deep) were located $1 \mathrm{~cm}$ behind the keys. Three solenoid food dispensers were placed on the roof of the alcove. When one of them was actuated it delivered about 4-6 grains of millet to the cup next to the relevant key. Stimuli were back-projected with an automatic projector and via an oblique front-silvered mirror, onto the pecking keys. The stimulus display on the center and side keys could be controlled with solenoid shutters. The stimuli (some are shown in Fig. 1) appeared on the keys as white abstract figures, each about $10 \mathrm{~mm}$ across, on a dark background. A microcomputer with an interface provided on-line control (Xia et al., 1991). 


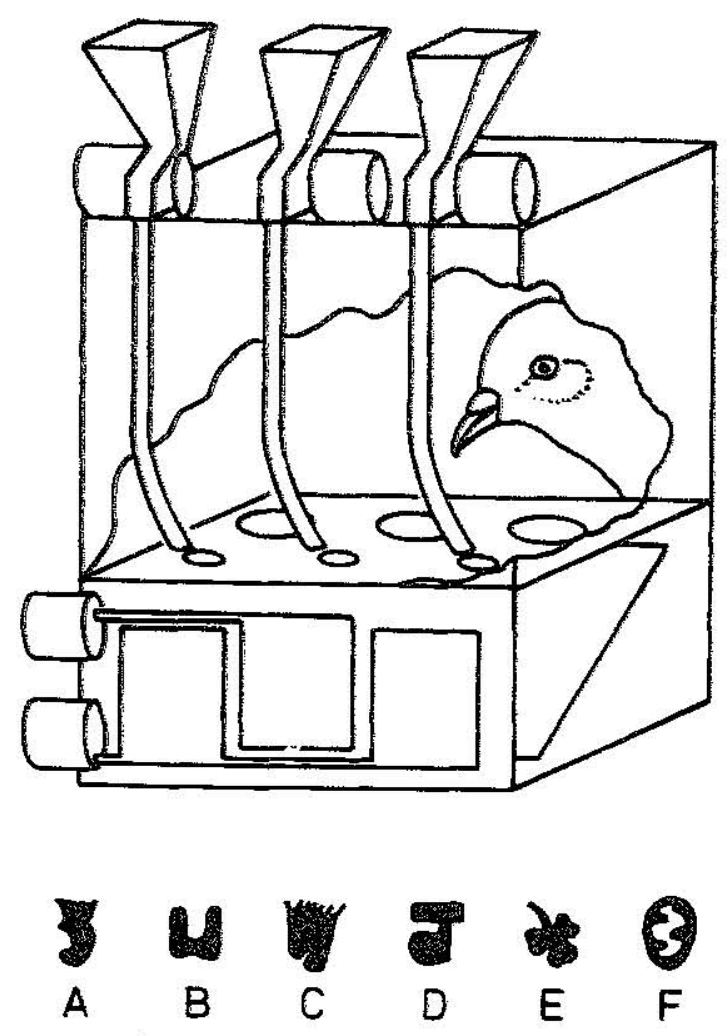

Fig. 1. Illustration of experimental set-up. Top: Chamber alcove with keys and feeders. Bottom: Stimulus patterns.

\section{Procedure}

The pigeons were first conditioned to peck the diffusely illuminated keys using an autoshaping procedure for six 30-min sessions. Then they were trained with a symbolic matching-to-sample procedure. Sessions were daily, except weekends. Each session consisted of the successive presentation of 32 stimulus configirations, each of which comprised a sample and two comparison patterns (Table 1, a). Each of the trials began with an intertrial interval of $6 \mathrm{~s}$ during which the houselight was on. The presentation of a sample stimulus on the center key followed. A response to this key was rewarded at the center cup. The comparison stimuli then appeared additionally on the two side keys. A response to the correct, equivalent comparison stimulus was rewarded at the corresponding side cup. Responding to the incorrect, non-equivalent stimulus led to a $4 \mathrm{~s}$ time-out during which the houselight was off. In the correction trial that followed, the same stimulus configuration was presented again. This procedure ceased when the subjects chose correctly.

During the initial training sessions the response requirement to the trial stimuli was gradually increased from an FR 1 to a modified $\mathrm{r} R \mathrm{R}$ requirement. The latter condition required that, after pecking eight times to the sample stimulus and obtaining reinforcement, the pigeon had to issue eight consecutive pecks to the correct comparison stimulus to obtain further reinforcement. If an intervening peck was delivered to the incorrect comparison stimulus, the correct peck count was reset to zero. Time-out followed eight 


\section{TABLE 1}

Stimulus configurations and average percent side choices during training (a) and tests (b, c) of Experiment I. A to F: stimulus patterns; $\rightarrow, \cdots$ : directional response biases explained in the text.

\begin{tabular}{ccclll}
\hline \multicolumn{2}{c}{ (a) Training. + : rewarded choice } & & & \\
No. & Choices & Left & Center & Right & Choices \\
1 & $9 \%$ & $\mathrm{E} \rightarrow$ & $\mathrm{A}$ & $\rightarrow \mathrm{B}+$ & $91 \%$ \\
2 & $6 \%$ & $\mathrm{E} \rightarrow$ & $\mathrm{C}$ & $\rightarrow \mathrm{B}+$ & $94 \%$ \\
3 & $2 \%$ & $\mathrm{~B} \rightarrow$ & $\mathrm{D}$ & $\rightarrow \mathrm{E}+$ & $98 \%$ \\
4 & $2 \%$ & $\mathrm{~B} \rightarrow$ & $\mathrm{F}$ & $\rightarrow \mathrm{E}+$ & $98 \%$ \\
5 & $95 \%$ & $+\mathrm{A} \leftarrow$ & $\mathrm{B}$ & $\leftarrow \mathrm{F}$ & $5 \%$ \\
6 & $86 \%$ & $+\mathrm{C} \leftarrow$ & $\mathrm{B}$ & $\leftarrow \mathrm{D}$ & $14 \%$ \\
7 & $91 \%$ & $+\mathrm{D} \leftarrow$ & $\mathrm{E}$ & $\leftarrow \mathrm{C}$ & $9 \%$ \\
8 & $91 \%$ & $+\mathrm{F} \leftarrow$ & $\mathrm{E}$ & $\leftarrow \mathrm{A}$ & $9 \%$ \\
9 & $24 \%$ & $\mathrm{E} \rightarrow$ & $\mathrm{B}$ & $\leftarrow \mathrm{A}+$ & $76 \%$ \\
10 & $32 \%$ & $\mathrm{E} \rightarrow$ & $\mathrm{B}$ & $\leftarrow \mathrm{C}+$ & $68 \%$ \\
11 & $19 \%$ & $\mathrm{~B} \rightarrow$ & $\mathrm{E}$ & $\leftarrow \mathrm{D}+$ & $81 \%$ \\
12 & $29 \%$ & $\mathrm{~B} \rightarrow$ & $\mathrm{E}$ & $\leftarrow \mathrm{F}+$ & $71 \%$ \\
13 & $74 \%$ & $+\mathrm{E} \rightarrow$ & $\mathrm{F}$ & $\leftarrow \mathrm{A}$ & $26 \%$ \\
14 & $81 \%$ & $+\mathrm{E} \rightarrow$ & $\mathrm{D}$ & $\leftarrow \mathrm{C}$ & $19 \%$ \\
15 & $74 \%$ & $+\mathrm{B} \rightarrow$ & $\mathrm{C}$ & $\leftarrow \mathrm{D}$ & $26 \%$ \\
16 & $63 \%$ & $+\mathrm{B} \rightarrow$ & $\mathrm{A}$ & $\leftarrow \mathrm{F}$ & $37 \%$
\end{tabular}

(b) Transitivity test. *: expected choice

$\begin{array}{rrrllr}17 & 85 \% & \mathrm{~F} \leftarrow & \mathrm{A} & \leftarrow \mathrm{C} * & 15 \% \\ 18 & 85 \% & \mathrm{~F} \leftarrow & \mathrm{C} & \leftarrow \mathrm{A} & 15 \% \\ 19 & 90 \% & * \mathrm{C} \leftarrow & \mathrm{A} & \leftarrow \mathrm{F} & 10 \% \\ 20 & 100 \% & * \mathrm{~A} \leftarrow & \mathrm{C} & \leftarrow \mathrm{F} & 0 \% \\ 21 & 90 \% & \mathrm{C} \leftarrow & \mathrm{D} & \leftarrow \mathrm{F} * & 10 \% \\ 22 & 90 \% & \mathrm{C} \leftarrow & \mathrm{F} & \leftarrow \mathrm{D} * & 10 \% \\ 23 & 100 \% & * \mathrm{~F} \leftarrow & \mathrm{D} & \leftarrow \mathrm{C} & 0 \% \\ 24 & 100 \% & * \mathrm{D} \leftarrow & \mathrm{F} & \leftarrow \mathrm{C} & 0 \%\end{array}$

(c) Novel sample test

\begin{tabular}{lrlllr}
$1 \mathrm{c} / 2 \mathrm{C}$ & $0 \%$ & $\mathrm{E} \rightarrow$ & $\mathrm{X}$ & $\rightarrow \mathrm{B}$ & $100 \%$ \\
$3 \mathrm{c} / 4 \mathrm{c}$ & $0 \%$ & $\mathrm{~B} \rightarrow$ & $\mathrm{X}$ & $\rightarrow \mathrm{E}$ & $100 \%$ \\
$5 \mathrm{c}$ & $100 \%$ & $\mathrm{~A} \leftarrow$ & $\mathrm{X}$ & $\leftarrow \mathrm{F}$ & $0 \%$ \\
$6 \mathrm{c}$ & $90 \%$ & $\mathrm{C} \leftarrow$ & $\mathrm{X}$ & $\leftarrow \mathrm{D}$ & $10 \%$ \\
$7 \mathrm{C}$ & $90 \%$ & $\mathrm{D} \leftarrow$ & $\mathrm{X}$ & $\leftarrow \mathrm{C}$ & $10 \%$ \\
$8 \mathrm{c}$ & $80 \%$ & $\mathrm{~F} \leftarrow$ & $\mathrm{X}$ & $\leftarrow \mathrm{A}$ & $20 \%$ \\
$9 / 13 \mathrm{c}$ & $40 \%$ & $\mathrm{E} \rightarrow$ & $\mathrm{X}$ & $\leftarrow \mathrm{A}$ & $60 \%$ \\
$10 / 14 \mathrm{c}$ & $30 \%$ & $\mathrm{E} \rightarrow$ & $\mathrm{X}$ & $\leftarrow \mathrm{C}$ & $70 \%$ \\
$11 / 15 \mathrm{c}$ & $40 \%$ & $\mathrm{~B} \rightarrow$ & $\mathrm{X}$ & $\leftarrow \mathrm{D}$ & $60 \%$ \\
$12 / 16 \mathrm{c}$ & $40 \%$ & $\mathrm{~B} \rightarrow$ & $\mathrm{X}$ & $\leftarrow \mathrm{F}$ & $60 \%$ \\
\hline
\end{tabular}

pecks (consecutive or non-consecutive) to the incorrect comparison stimulus. A trial was scored as correct or incorrect, depending on whether it yielded reward or time-out; correction trials were disregarded. 
Tests

After the pigeons had completed the 110 prescheduled acquisition sessions they were familianzed with the extinction procedure that was to be used during test sessions. Accordingly, in ten additional sessions there was an increasing number of randomly selected trials (tour initially, ten finally) on which choices were neither rewarded nor punished. Completion of a simple FR 8 on a given side key led directly to the inter-trial interval. The actual test sessions that then followed consisted of the normal 32 training trials (rewarded or punished depending on the animal's responses) but in addition there were two, four or eight randomly interspersed trials with special stimulus configurations (Table $T, b$ and $c)$. On these trials choices of lateral stimuli were not reinforced. Each test configuration was presented on ten trials distributed over between ten and 40 test sessions, depending on the number of stimulus configurations involved in the particular test.

\section{Results}

\section{Training}

This phase involved the reinforced presentation of 16 stimulus configurations assembled from patterns $A$ to $F(T a b l e ~ 1, a)$. Each configuration was presented twice per session in a quasi-random order. Half of the configurations (Table 1,a: nos. 1,2,5,6,9,10,15 and 16) were designed to condition the pigeons to the equivalences $A=B$ and $B=C$. The remaining configurations were intended to condition the subjects to the equivalences $D=E$ and $E=F$. The learning task proved to be difficult for the pigeons. Five out of the six original subjects showed some learning, but only two of them actually reached the prescribed performance criterion within the pre-established training period. An average of $80 \%$ or more correct comparison choices during the last ten training sessions were deemed a necessary baseline for a meaningful testing of transitive responding. The results that are henceforth reported thus concern only the two successful birds.

The choice behavior of both pigeons during the criterion sessions concurred closely across all 16 training configurations $(r=0.79, F(1,14)=23.3, P<0.05)$. It is therefore valid to report pooled data. The overall average percentage of trials correct across all stimulus combinations was $83 \%$. Though there was some variation amongst configurations (Table 1,a) all choice correct scores were above the $50 \%$ chance level.

\section{Transitivity test}

Since the two pigeons appeared to have learned the equivalences $A=B, B=C, D=E$ and $E=F$ underlying the training configurations, it was now expected that they would also treat as equivalent the stimulus pairs $\mathrm{A}-\mathrm{C}$ and $\mathrm{D}-\mathrm{F}$. This was tested with eight novel configurations (Table 1,b). The pigeons chose according to the transitivity hypothesis on only half of the configurations (nos. 19,20,23 and 24). On the other four combinations (nos. 17,18,21, and 22) they preferred the non-equivalent, wrong comparison stimulus. There was thus no evidence of equivalence transitivity *. Both birds, in fact, quite

\footnotetext{
* While this experiment did not demonstrate equivalence transitivity it would be premature to deny such a capability to pigeons. The present failure was inevitable since, as eventually demonstrated, the subjects did not really learn the essential preliminary symbolic matching tasks. It is possible that a fully balanced training design (including both the configurations 1 to 16 , Table 1 and the symmetric configurations 1e to $16 \mathrm{e}$, Table 2) could yield success. In a different experiment it was demonstrated that pigeons can exhibit transitivity with respect to inequality relations (Fersen et al., 1991).
} 
consistently chose the left comparison stimuli, at a mean rate of $92 \%$ across all test trials. Though this could reflect a chance side bias emerging as a consequence of complete stimulus control loss, a more conservative hypothesis was provisionally entertained. The result might have arisen because only the training sample stimuli had not gained sufficient control over the pigeons' choice behavior.

\section{Novel sample test}

In order to check this possibility the center stimuli of the training configurations were replaced by novel patterns never previously seen by the pigeons (collectively denominated $X$ in Table 1,c). In spite of the novel center stimuli the first six configurations (nos. 1c to 8c; in the absence of meaningful center stimuli, configurations $1 \mathrm{c}$ to $4 \mathrm{c}$ collapse to two identical configurations) were treated much as the corresponding training configurations had been (a mean 95\% trials 'correct' as compared with 93\%). This indicates that here the center stimuli played little or no role in the original configurations. The remaining four test configurations (nos. 9c to 16c; they collapse to four configurations) on the other hand, did not elicit clear choice preferences (maximally $70 \%$ to any given side), indicating that responding to the corresponding original, well trained configurations (Table 1,a, nos. 9 to 16) must, after all, have been at least partly controlled by the center stimuli.

Regardless of that, some test configurations yielded a clear-cut right side preference (nos. 1c to $4 \mathrm{c}$ ) and overall all of them produced a slight right side advantage (average $59 \%$ choices). This supports the view that the predominance of left side choices observed in the previous test was not simply due to a chance side preference elicited by meaningless configurations.

\section{Novel comparison tests}

For reasons of symmetry it was now desirable to check the key role of lateral stimuli. Six configurations, containing the patterns $A$ to $F$ as central stimuli and stimuli novel to the pigeons as lateral stimuli, were inserted in what was planned to be a series of 12 test sessions. Neither pigeon completed more than the first session, however. The tests had to be abandoned because the subjects would not fulfill the response requirements to the test configurations, even when given extensive time. This 'result' underlines the relative importance of the habitual lateral stimuli in controlling side-key choices.

\section{Discussion}

At this point it seemed certain that, although the subjects had not shown transitive respondir $\mathrm{y}$ as originally expected, they were not behaving according to chance either. At least the lateral stimuli were controlling choices but what were the precise rules that the pigeons were obeying?

A close examination of the training set (Table 1,a) eventually suggested an hypothesis based on side biases arising from a subtle imbalance. When serving as samples the six patterns composing them were not involved in any design peculiarities. They were always first reinforced centrally and subsequently each of them was as often associated with reinforcement on the right side as on the left side. When, however, the patterns served as comparison stimuli, there was a hidden design asymmetry. In patterns $B$ and $E$ on the one hand, each occurred eight times as lateral stimuli, two times on the right and six times on the left. On six of the same eight instances though, reinforcement was scheduled on the 
right side, only on two instances on the left. $A$ bias to respond to the right side in the presence of B and E could be expected. Patterns A, C, D and F, on the other hand, each occurred as comparisons in four training configurations, three times on the right and once on the left key. In only one of these four configurations, however, was reinforcement scheduled on the right side, and in three configurations on the left side. Accordingly the $A$, C, D and F patterns could be expected to elicit a left response bias. Note though that the directing effects of all six stimuli arose only because of their excessive frequency on the non-reinforced sides of the configurations **; see also *.

The vectors inserted throughout Table 1 symbolize the hypothesized side directing effects of lateral stimuli. Examination reveals that when the vectors point to the same side, they do so to the side preferentially chosen by the subjects. When the vectors point in opposite directions the subjects' preferences are indeed generally more balanced, although there are exceptions (particularly within the training configurations).

\section{Experiment ॥}

The explanation now offered for the results of Experiment $I$ is that the six stimuli composing the training configurations had each acquired a specific response-directing property. These were expressed when the stimuli were present on the side keys and had additive properties. Though partly supported by the preceding data, the hypothesis clearly required independent confirmation. The tests incorporated in the experiment now described were designed to provide the necessary information.

\section{Method}

The same subjects and apparatus as in the preceding experiment were used. While the training procedure continued as before, a series of new stimulus arrangements were tested under the same extinction conditions described earlier.

\footnotetext{
* In a preliminary account based only on part of the evidence presented here, Fersen and Emmerton (1987) considered the possibility that balanced side stimulus bias configurations might lead to a subsidiary evaluation of the bias signalled by the center stimulus. That hypothesis can now be dismissed. It should have led to a right key preference in balanced bias test configurations containing $B$ or $E$ as central stimuli (nos. $1 \mathrm{~d}$ to $4 \mathrm{~d}, 9 \mathrm{~d}$ to $12 \mathrm{~d}$ and $9 \mathrm{e}$ to $12 \mathrm{e}$ ) and to a left preference in such configurations that include patterns $A, C, D$, or $F$ as central elements ( $5 \mathrm{~d}$ to $8 \mathrm{~d}, 13 \mathrm{~d}$ to $16 \mathrm{~d}$ and $13 \mathrm{e}$ to $16 \mathrm{e}$ ). This did not happen to any significant degree (average choices in the required sense only $56 \%$ ). In the same report it was also considered that stimuli $B$ and $E$ might have induced a stronger (right) side bias than stimuli $\mathrm{A}, \mathrm{C}, \mathrm{D}$ and $\mathrm{F}$ a (left) side bias due to their different frequency of occurrence as lateral stimuli in the training configurations (on eight and four opportunities respectively). This should have led to an overall right side preference across the balanced bias configurations just enumerated but in fact on average only $47 \%$ of the pertinent choices were to the right. There is thus no basis for assuming bias strength differences.

** That non-reinforced visual stimuli can exert an unexpectedly strong response control was also established in a different experiment with pigeons using a similar apparatus and procedure (Fersen and Delius, 1989).
} 


\section{Results}

The average percent side-key choices generated by the pigeons are listed in Table 2 , along with the test configurations employed. The choices predicted by the directional response bias hypothesis are indicated by the previously explained vectors.

\section{Sample / comparison exchange test}

Sixteen test configurations were produced by interchanging the sample stimuli with the incorrect comparison stimuli of the original training configurations (sample/correct comparison exchanges were already part of the training set). All configurations involved opposite response bias vectors. In accordance with this fact no clear choice paftern emerged and only two configurations yielded preference scores above $70 \%$ (Table 2,d).

\section{Comparison exchange test}

As the above condition tested the hypothesis only in a negative fashion, a further set of new test configurations was assembled by exchanging the side stimuli of the training configurations. In accordance with the equi-directional vectors that corresponded to them, high scores were attained with the first eight configurations (nos. 1e to $8 \mathrm{e}$, both vectors poirting to same side; average $91 \%$ choices according to expectation; Table 2,e). The remaining eight configurations (nos. $9 \mathrm{e}$ to $16 \mathrm{e}$, vectors pointing in opposite directions), yielded low preference scores (none exceeding $65 \%$, compatible with random responding), again in accordance with our expectaticns. At the same time, the results of this test were definitely incompatible with generalized symbolic matching behavior (only $25 \%$ of choices were compatible; see footnote on page 143).

\section{Same comparison test}

As a further check two pairs of patterns known to the pigeons and symbolizing different response tendencies ( $E, B$ to the right; $C, D$ to the left) were assembled into yet another four novel test configurations. One pattern was presented on the center key and the other pattern was shown on both lateral keys (Table 2,f). According to the side bias hypothesis, two of these test configurations (nos. 25 and 26) should yield a right key preference while the two others (nos. 27 and 28) should produce a right key preference (Table 2,f, see vectors). Indeed, the first pair resulted in an average $94 \%$ right choices and the latter pair yielded $100 \%$ left choices.

\section{Single stimulus test}

This test was meant as a further check whether the central stimuli were of any consequence. The same stimulus projected on all three keys would perhaps produce a maximal side "ey preference. Six configurations of this kind were composed from each of the original patterns. All the configurations produced choices according to the directional hypothesis (Table 2,g). The configurations $29,30,32$ and 33 correspond to configurations $26,25,27$ and 28 respectively of the previous test (Table 2,f), except for the different central stimuli. The results, however, concur (average choices respectively $96 \%$ and $97 \%$ ), confirming that the center elements of test configurations were indeed irrelevant (see footnote on page 145).

\section{New configurations test}

For this final test, four (strictly speaking 16) test configurations were constructed by combining the familiar stimuli in yet other novel ways. Two different patterns served as 
comparison stimuli, while each one of the remaining four patterns appeared as the central stimulus (labelled with $Y$ in Table 2,h). Since both lateral patterns were associated in each case with a left response bias, it was expected that the pigeons would choose predomi-

\section{TABLE 2}

Stimulus configuzations used and average percent side choices obtained during tests $\boldsymbol{d}$ to $\boldsymbol{h}$ (Experiment 11). A to F: stimuliss patterns; $\rightarrow$. : directional response biases explained in the text.

\begin{tabular}{|c|c|c|c|c|c|}
\hline \multicolumn{6}{|c|}{ (d) Sample/ comparison exchange test } \\
\hline No. & Choires & left & Center & Right & Choices \\
\hline $1 d d$ & $55 \%$ & $A \leftarrow$ & E & $\rightarrow B$ & $45 \%$ \\
\hline $2 d$ & $65 \%$ & $c \leftarrow$ & E & $\rightarrow \mathrm{B}$ & $35 \%$ \\
\hline $3 d$ & $70 \%$ & $\mathrm{D} \leftarrow$ & $B$ & $\rightarrow E$ & $30 \%$ \\
\hline $4 d$ & $60 \%$ & $F \leftarrow$ & B & $\rightarrow \mathrm{E}$ & $40 \%$ \\
\hline $5 d$ & $65 \%$ & $A \leftarrow$ & $F$ & $\rightarrow \mathrm{B}$ & $35 \%$ \\
\hline $6 d$ & $60 \%$ & $C \leftarrow$ & $\mathrm{D}$ & $\rightarrow \mathrm{B}$ & $40 \%$ \\
\hline $7 d$ & $55 \%$ & $\mathrm{D} \leftarrow$ & C & $\rightarrow E$ & $45 \%$ \\
\hline $8 d$ & $45 \%$ & $F \leftarrow$ & A & $\rightarrow E$ & $55 \%$ \\
\hline $9 \mathrm{~d}$ & $20 \%$ & $B \rightarrow$ & E & $\leftarrow A$ & $80 \%$ \\
\hline $10 \mathrm{~d}$ & $50 \%$ & $\mathrm{~B} \rightarrow$ & $E$ & $\leftarrow C$ & $50 \%$ \\
\hline $11 d$ & $50 \%$ & $E \rightarrow$ & B & $\leftarrow D$ & $50 \%$ \\
\hline $12 d$ & $50 \%$ & $E \rightarrow$ & B & $\leftarrow F$ & $50 \%$ \\
\hline $13 d$ & $85 \%$ & $E \rightarrow$ & A & $\leftarrow F$ & $15 \%$ \\
\hline $14 d$ & $70 \%$ & $E \rightarrow$ & $C$ & $\leftarrow D$ & $30 \%$ \\
\hline $15 d$ & $50 \%$ & $B \rightarrow$ & D & $\leftarrow C$ & $50 \%$ \\
\hline $16 d$ & $70 \%$ & $B \rightarrow$ & $\mathrm{F}$ & $\leftarrow A$ & $30 \%$ \\
\hline
\end{tabular}

(e) Comparison exchange test. *: expected symbolic matching choices

\begin{tabular}{cccccr}
$1 e$ & $5 \%$ & $* \mathrm{~B} \rightarrow$ & $\mathrm{A}$ & $\rightarrow \mathrm{E}$ & $95 \%$ \\
$2 \mathrm{e}$ & $0 \%$ & $* \mathrm{~B} \rightarrow$ & $\mathrm{C}$ & $\rightarrow \mathrm{E}$ & $100 \%$ \\
$3 \mathrm{e}$ & $20 \%$ & $* \mathrm{E} \rightarrow$ & $\mathrm{D}$ & $\rightarrow \mathrm{B}$ & $80 \%$ \\
$4 \mathrm{e}$ & $10 \%$ & $* \mathrm{E} \rightarrow$ & $\mathrm{F}$ & $\rightarrow \mathrm{B}$ & $90 \%$ \\
$\mathrm{Se}$ & $95 \%$ & $\mathrm{~F} \leftarrow$ & $\mathrm{B}$ & $\leftarrow \mathrm{A}^{*}$ & $5 \%$ \\
$6 \mathrm{e}$ & $85 \%$ & $\mathrm{D} \leftarrow$ & $\mathrm{B}$ & $\leftarrow \mathrm{C} *$ & $15 \%$ \\
$7 \mathrm{e}$ & $90 \%$ & $\mathrm{C} \leftarrow$ & $\mathrm{E}$ & $\leftarrow \mathrm{D} *$ & $10 \%$ \\
$8 \mathrm{e}$ & $95 \%$ & $\mathrm{~A} \leftarrow$ & $\mathrm{E}$ & $\leftarrow \mathrm{F}$ & $5 \%$ \\
$9 \mathrm{e}$ & $40 \%$ & $* \mathrm{~A} \leftarrow$ & $\mathrm{B}$ & $\leftarrow \mathrm{E}$ & $60 \%$ \\
$10 \mathrm{e}$ & $40 \%$ & $* \mathrm{C} \leftarrow$ & $\mathrm{B}$ & $\rightarrow \mathrm{E}$ & $60 \%$ \\
$11 \mathrm{e}$ & $55 \%$ & $* \mathrm{D} \leftarrow$ & $\mathrm{E}$ & $\rightarrow \mathrm{B}$ & $45 \%$ \\
$12 \mathrm{e}$ & $40 \%$ & $* \mathrm{~F} \leftarrow$ & $\mathrm{E}$ & $\rightarrow \mathrm{B}$ & $60 \%$ \\
$13 \mathrm{e}$ & $60 \%$ & $\mathrm{~A} \leftarrow$ & $\mathrm{F}$ & $\rightarrow \mathrm{E}$ & $40 \%$ \\
$14 \mathrm{e}$ & $65 \%$ & $\mathrm{C} \leftarrow$ & $\mathrm{D}$ & $\rightarrow \mathrm{E} *$ & $35 \%$ \\
$15 \mathrm{e}$ & $55 \%$ & $\mathrm{D} \leftarrow$ & $\mathrm{C}$ & $\rightarrow \mathrm{B} *$ & $45 \%$ \\
$16 \mathrm{e}$ & $65 \%$ & $\mathrm{~F} \leftarrow$ & $\mathrm{A}$ & $\rightarrow \mathrm{B} *$ & $35 \%$ \\
(f) Same comparison tesi & & & & \\
25 & $13 \%$ & $\mathrm{~B} \rightarrow$ & $\mathrm{E}$ & $\rightarrow \mathrm{B}$ & $87 \%$ \\
26 & $0 \%$ & $\mathrm{E} \rightarrow$ & $\mathrm{B}$ & $\rightarrow \mathrm{E}$ & $100 \%$ \\
27 & $100 \%$ & $\mathrm{C} \leftarrow$ & $\mathrm{D}$ & $\leftarrow \mathrm{C}$ & $0 \%$ \\
28 & $100 \%$ & $\mathrm{D} \leftarrow$ & $\mathrm{C}$ & $\leftarrow \mathrm{D}$ & $0 \%$ \\
\hline
\end{tabular}


TABLE 2 (continued)

\begin{tabular}{lrllll}
\hline (g) Single stimulus test & & & \\
29 & $0 \%$ & $\mathrm{E} \rightarrow$ & $\mathrm{E}$ & $\rightarrow \mathrm{E}$ & $100 \%$ \\
30 & $25 \%$ & $\mathrm{~B} \rightarrow$ & $\mathrm{B}$ & $\rightarrow \mathrm{B}$ & $75 \%$ \\
31 & $100 \%$ & $\mathrm{~A} \leftarrow$ & $\mathrm{A}$ & $\leftarrow \mathrm{A}$ & $0 \%$ \\
32 & $100 \%$ & $\mathrm{C} \leftarrow$ & $\mathrm{C}$ & $-\mathrm{C}$ & $0 \%$ \\
33 & $100 \%$ & $\mathrm{D} \leftarrow$ & $\mathrm{D}$ & $\leftarrow \mathrm{D}$ & $0 \%$ \\
34 & $100 \%$ & $\mathrm{~F} \leftarrow$ & $\mathrm{F}$ & $-\mathrm{F}$ & $0 \%$ \\
(h) New configurations. Y: one of the four remaining training stimuli & \\
35 & $95 \%$ & $\mathrm{~A} \leftarrow$ & $\mathrm{Y}$ & $\leftarrow \mathrm{D}$ & $5 \%$ \\
36 & $100 \%$ & $\mathrm{~A} \leftarrow$ & $\mathrm{Y}$ & $\leftarrow \mathrm{C}$ & $0 \%$ \\
37 & $95 \%$ & $\mathrm{~F} \leftarrow$ & $\mathrm{Y}$ & $\leftarrow \mathrm{D}$ & $5 \%$ \\
38 & $100 \%$ & $\mathrm{~F} \leftarrow$ & $\mathrm{Y}$ & $\leftarrow \mathrm{C}$ & $0 \%$ \\
\hline
\end{tabular}

nantly the left key. In fact the pigeons strongly preferred that side (average $97 \%$ ) and were clearly not disturbed by the varying center stimuli.

\section{General Discussion}

Summarizing the results of both experiments, of all 28 test configurations of the balanced right/left bias type, only two (Table 2: 9d, 13d) led to a side preference stronger than $70 \%$. Overall these configurations on average led to an almost even $48 \%$ right and $52 \%$ left side choices, precisely as demanded by the directional bias hypothesis. All remaining test configurations involved additive side biases to the right or to the left. On average, all ten test trials involving additive biases to the right led to $94 \%$ right key choices, and all 26 in which a left bias was expected did indeed lead to $94 \%$ left key choices. None of these 36 test configurations yielded choice preferences that contravened the predictions of the directional bias hypothesis.

Thus, of all 64 novel configurations shown under extinction conditions, 62 can be contidently considered as having yielded choice behavior in agreement with the hypothesis that the pigeons chose side keys solely in response to lateral stimuli according to the rules: if stimulus $B$ (or $E$ ) go right; if stimulus $A$ (or $C$ or $D$ or $F$ ) go left ( $P<0.001$, binomial test). Since none of the test configurations were ever reinforced it must mean that the response rules were ar yuired with, and maintained by, the 16 regularly reinforced training configurations. The mechanism by which this came about has been expounded in connection with the discussion of the results for Experiment 1.

However, the responding to some of the training constellations requires a special explanation going beyond the side bias hypothesis. With the balanced bias training configurations the pigeons cannot have chosen sides the way they did just on the basis of the lateral stimuli: configurations nos, 9 to 12 (Table $1, a$, average $74 \%$ correct right choices) are identical regarding these stimuli to configurations nos. 13 to 16 respectively (average $73 \%$ correct left choices; compare also Table $1, \mathrm{C}$, nos. $9 \mathrm{c} / 13 \mathrm{c}$ to $12 \mathrm{c} / 16 \mathrm{c}$ ). These subgroups differ only with respect to the center stimuli (B or E vs. A, C, D or F). Although the effect is weak icompare the average $93 \%$ correct choices to configurations 
nos. 1 8 8), these stimuli must have intuenced choices by rules additional to those assumed to control the responses to test configurations. Both the muttiple rule model ta set of rules of the type "given sample $M$ choose comparison $N^{\prime}$ ) and the configurational models (each three-stimulus constellation controls side choice as a compound) of Carter and Werne $(1978)$ could be relevant here. Since the subjects did not reveal definite reversed key preferences then the hateral stimulit of the relevant training configurations were side excharged (Table 2,e, nos. $9 e$ to 16e), as they should have done if the multiple rule model applied, thanining constellations according to the configurational model (effectively a rote-learning of individual conffigurations; see also footnote on page 145). It cannot be excluded that they also partially dea dit in the same way with the remaining training configurations (nos. 1 to 8), but generally they must have relied predominantly on the directional rules that were absolutely dominam in the various interspersed unreinforced tests where rote learning was prectuded.

能 is instructive to consider how the pigeons must actually have behaved according to the ohrectional oules when rote memory information was not applicable. After having responded to the center key the subjects would initially tum randomly to the right or left key $\left(P_{r}=P_{1}=0.5\right)$. Pf the key examined at any time displayed a 'this-side' stimulus the subject would choose that key (absorbing state) with a high probability $\left(P_{\mathrm{tc}}\right)$ and only switch to examine the opposite key (transition state) with a low probability $\left(P_{\mathrm{ts}}=1-P_{\mathrm{tc}}\right)$. If the key examined at any time displayed an 'other-side' stimulus the subject would choose that key (absorbing state) with only a low probability $\left(P_{o c}\right)$ but switch to examine the opposite key (transition state) with a high probability $\left(P_{\text {os }}=1-P_{o c}\right)$. Stochastic simulation runs based on this model showed that common values of 0.85 for the higher probabilities $\left(P_{\mathrm{tc}}=P_{\mathrm{os}}\right)$ and 0.15 for the lower probabilities $\left(P_{\mathrm{ts}}=P_{\text {oc }}\right)$ yielded a choice score array that is closely similar to that empirically found with the test configurations. Remark that there are two kinds of ballanced bias configurations: some where both lateral stimuli instruct a 'this-side' response $(\leftarrow, \rightarrow)$ and others where both lateral stimuli instruct an 'other-side' response $(\rightarrow, \leftarrow)$. It was noticed that pigeons would, on occasion, repeatedly examine both lateral stimuli before making a choice but it was not recorded which particular configurations triggered this behavior. The simulation indicates that such vacillation should have been caused matury by the double 'other-side' configurations (an average of about 7.1 side-switches as compared to about 1.6 with the other configurations; see also *).

The Markovian choice mechanism proposed is in fact similar to that which Wright (1990) attributes to pigeons solving conventional matching-to-sample tasks and which he supports with actual quantitative data on key inspections. The present experiment suggests a procedure with which the model could be further verified. A systematization of its design would supply a more direct control over the response location than that provided by the varying levels of stimulus discriminability to which Wright resortcd. Such a design could dispense with the central stimuli and be based on a simple conditional two-key paradigm

\footnotetext{
* Double 'other-side' configurations, could accordingly be: expected to yieid particularly long choice times. These were not measured. However, it is striking that the variability of choice percentages was higher with them than with the double 'this-side' configurations (mean deviation from average $8.2 \%$, $n=12$ versus $4.0 \%, n=16 ; P<0.01$, Mann-Whitney test). To model this effect the simulation has to include an additional side-switching truncation parameter that causes choices by default.
} 
where the programmed left-right distribution of teinforced responses would be a gioint function of the stimuli presented on both keys. It is likely, though, that the naturalistic spatial contiguity of correct response and reward delivery, instrumented by the apparatus used here, will turn out to be important for the development of stimulus-dependent directional biases of the kind needed. The general technique of variable reinforcement location may prove more universally useful for the study of the effect of spatial stimulus/ response/reward contingency, or co-location variations that have witherto been rather neglected in behavior-analytic studies.

\section{Acknowledgements}

The research was supported by grants from the Deutsche Forschungsgemeinschaft. We thank Prof. S.E.G. Lea (University of Exeter, UK) for several useful suggestions during the preparation of this article and A. Niemuth for excellent help with the pieparation of the manuscript.

\section{References}

Carter, D.E. and Werner, T.J. 1978. Compl $\times x$ learning and information processing by pigeons: a critical analysis. J. Exp. Anal. of Behav., 29: 565-601.

Fersen, L. von and Delius, J.D. 1989. Long term retention of many vistal patterns by pigeons. Ethology, 82: 141-155.

Fersen, L von and Emmerton, J. 1987. Valorización direccional de estimulos por palomas en una discriminación condicional. Revista Mexicana de Análisis de la Conducta, 13: 273-285.

Fersen, L. von, Wynne, C.D.L., Delius, I.D. and Staddon, J. E.R. 1991. Transitive inference formation in pigeons. J. Exp. Psychol.: Anim. Behav. Process. 17: 334-341.

Sidman, M. 1990. Equivalence relations: Where do they come from? In: D F. Blackman and $H$. Lejeune (Editors), Behavior Analysis in Theory and Practice, Contributions and Controversies. Hove, Erlbaum, pp. 93-114.

Wright, A.A. 1990. Markov choice processes in simultaneous matching-tu-sample at different levels of discriminability. Anim. Learn. Behav., 18: 277-286.

Xia, L., Wynne, C.D.L., Münchow-Pohl, F. von and Delius, J. D. 1991. Psychobasic: a Basic dialect for the control of psychological experiments with the Commodore-64 and Dela interfacing. Behav. Res. Meth., Instrum. Comp., 23: 72-76. 